\title{
Efficacy of sticky and standard ovitraps for Aedes aegypti in Trinidad, West Indies
}

\author{
Dave D. Chadee ${ }^{1 \otimes}$ and Scott A. Ritchie ${ }^{2,3}$ \\ ${ }^{1}$ Department of Life Sciences, University of the West Indies, St. Augustine, Trinidad, West Indies \\ ${ }^{2}$ School of Public Health, Tropical Medicine and Rehabilitation Sciences, James Cook University, Cairns, Australia \\ ${ }^{3}$ Tropical Population Health Services, Queensland Health, Cairns, Australia
}

Received 22 March 2010; Accepted 24 August 2010

\begin{abstract}
The double sticky trap (DST) is described for the first time and is evaluated along with standard ovitraps and sticky traps (STs) to determine population densities of Ae. aegypti in the urban township of St. Augustine and the rural community of Tamana, Trinidad, West Indies. Ten houses were selected at each study site. At each of the ten houses, one ovitrap, one ST, and one DST were placed using the criteria established for placement of ovitraps. The results showed the three trapping methods successfully collected Ae. aegypti mosquitoes. All three traps collected significantly more adults or eggs in St. Augustine than in Tamana. DSTs collected 2,286 adults from St. Augustine vs 316 adults from Tamana ( $<<0.002)$, STs collected 1,480 and 220 adults, respectively ( $<<0.01$ ), and the ovitraps collected 2,735 and 517 eggs, respectively from St. Augustine and Tamana $(\mathrm{p}<0.002)$. Based on these results, the DSTs collected significantly $(\mathrm{P}<0.02)$ more adults than the STs. The DSTs and STs collected both adult and immature stages which can be used for toxicology, virology, and PCR studies and are suitable alternative Ae. aegypti surveillance tools for the Caribbean and Latin American region. Journal of Vector Ecology 35 (2): 395-400. 2010.
\end{abstract}

Keyword Index: Ae. aegypti, surveillance tools, sticky traps, double sticky traps, ovitraps, Trinidad.

\section{INTRODUCTION}

In the early $20^{\text {th }}$ century, attractants of biological or microbiological origin found at oviposition substrates, especially decomposing organic matter, were found to be attractive to Aedes aegypti L. (Buxton and Hopkins 1927, Manefield 1951, O’Gower 1963). Gjullin et al. (1965) reported that grass infusion and log pond water increased oviposition by Ae. aegypti and Culexpipiens quinquefasciatus Say. However, Hazard et al. (1967) showed Ae. aegypti was not attracted to the odor of hay infusion in an olfactometer. Despite these studies, infusions using various botanicals have been integrated into conventional ovitraps made from black jars containing tap water that were attractive to gravid female mosquitoes (Fay and Eliason 1966). The combined effect of black ovitraps and hay infusions was reported to remove large portions of the mosquito population (Reiter et al. 1991, Trexler et al. 1998), but Chadee et al. (1993) reported the failure of this combination to elicit increase in oviposition and this was later supported by others who determined that the failure resulted from changes in bacterial activity, especially fermentation (Santana et al. 2006).

Another "new" tool, the lethal trap, has re-emerged with old generation technology, that is, the Adhesive Pest Management (APM) approach which uses the sticky fly paper concept developed in 1880 by the Tanglefoot Company (Grand Rapids, MI). using adhesive paper to line ovitraps containing plain tap water. These traps are already attractive to gravid females (Fay and Eliason 1966) but collect mosquitoes when they alight on the sticky substance before, during, or after oviposition (Muir and Kay 1998,
Ordonez-Gonzalez et al. 2001). Modifications include adding mouse odor (McCall et al. 1996), using velour paper strips treated with synthetic pyrethroids, filling with hay infusion (Zeichner and Perich 1999), and adding synthetic oviposition attractants (Santana et al. 2006). Ritchie et al. (2003), using knowledge of the life history, ecology, and behavior of Ae. aegypti mosquitoes, modified the sticky trap to perform as an adulticiding trap and demonstrated its utility in epidemiological studies.

In the Caribbean region, Ae. aegypti surveillance is based primarily on house-to-house larval inspections of artificial and natural breeding containers (drums, buckets, basins, tree-holes, etc.) (PAHO 1994) and on modified ovitraps (Fay and Eliason 1966) which determine the presence or absence of the immature stages of the mosquitoes, mainly eggs (PAHO 1994, Chadee 2009), though more recently the pupal stage has been used as a proxy for adults (Focks and Chadee 1997). In 2008, a major outbreak of dengue fever occurred in Trinidad during the latter part of the rainy season with an incidence rate of 182 cases per 100,000 (Chadee 2010). During this outbreak, Insect Vector Control Division, Ministry of Health embarked on an island-wide mosquito control program, employing space-spraying, internal residual spraying, focal application of insecticides and community based source reduction programs (Chadee 2010). As an adjunct strategy, two sticky trap types, previously used in Australia were made available and were tested as a surveillance tool in housing areas in Trinidad.

The objective of this study was to compare the efficacy of standard ovitraps with that of two types of sticky traps, namely the sticky ovitrap (ST) and double sticky trap (DST), for monitoring adult mosquito populations in the urban 
center of St. Augustine and in the rural village of Tamana, Trinidad, West Indies.

\section{MATERIALS AND METHODS}

\section{Study sites}

This study was conducted for ten weeks at two sites: St. Augustine $\left(10^{\circ} 38^{\prime} \mathrm{N} ; 60^{\circ} 23^{\prime} \mathrm{W}\right)$, an urban university town with 3,000 houses and approximately 15,000 people, and Tamana, a rural community of $\left(10^{\circ} 49^{\prime} \mathrm{N} ; 61^{\circ} 19^{\prime} \mathrm{W}\right)$, nestled in the forested foothills of the Central Range located in eastcentral Trinidad with 80 houses and 300 people.

Trinidad traditionally experiences two seasons, the wet season occurring between June and December and the dry season from January to May of each year. The average temperature ranges from $22^{\circ} \mathrm{C}$ to $30.5^{\circ} \mathrm{C}$, but it is generally hotter in the wet season than in the dry season. This study was conducted during the wet season from October to December, 2008.

\section{Trapping methods}

Aedes aegypti oviposition was monitored using modified ovitraps (Fay and Eliason 1966) as described by Chadee and Corbet (1987). Each ovitrap consisted of a black, cylindrical, glass jar (height $13 \mathrm{~cm}$, diameter $6 \mathrm{~cm}$ ) containing about $375 \mathrm{ml}$ of tap water and a removable "paddle," a thin strip of brown hardboard $(12.5 \mathrm{~cm} \times 2.5$ $\mathrm{cm}$ ), on which the mosquitoes laid eggs just above the water level. There was no overflow hole in the side of the jar.

The ST was similar to that used in north Queensland, Australia (Ritchie et al. 2003) and consisted of a 1.2 liter black golf bucket with a $21.5 \times 5.5 \mathrm{~cm}$ plastic strip coated in polybutylene adhesive (UVR 32, Atlantic Paste and Glue, Brooklyn, NY) fastened to the opposite inner wall of the bucket with $50 \mathrm{~mm}$ paper clips (Figure 1). The trap was filled with aged tap water $(500 \mathrm{ml})$ to the level of the adhesive strips. Black plastic mesh $(1.7 \times 1.7 \mathrm{~cm}$ holes $)$ was secured over the bucket to minimize vertebrate contact with the glue. A single compressed lucerne (alfalfa) pellet (0.5 g) was added to the water, because it has been shown to increase Ae. aegypti oviposition in ovitraps (Ritchie 2001).

The DST consisted of a 1.2 liter black golf bucket with two plastic panels coated in polybutylene adhesive, sitting flush against each other against the inner surface or wall of the bucket. The top half of the trap was made of a similar 1.2 liter black golf bucket with the bottom removed. The walls of the bottomless bucket were also lined with two glue panels as described above. The other bucket containing sticky panels was inverted over the first and fastened together using two "fold back" clips to clamp the buckets together (Figure 1). The trap was filled with aged tap water $(350 \mathrm{ml})$ and a $0.5 \mathrm{~g}$ lucerne compressed pellet added to the water but no mesh cover was applied. This trap is described for the first time.

\section{Trap deployment and servicing}

At ten different houses in Tamana and St. Augustine, the traps were placed as follows at each house: one conventional

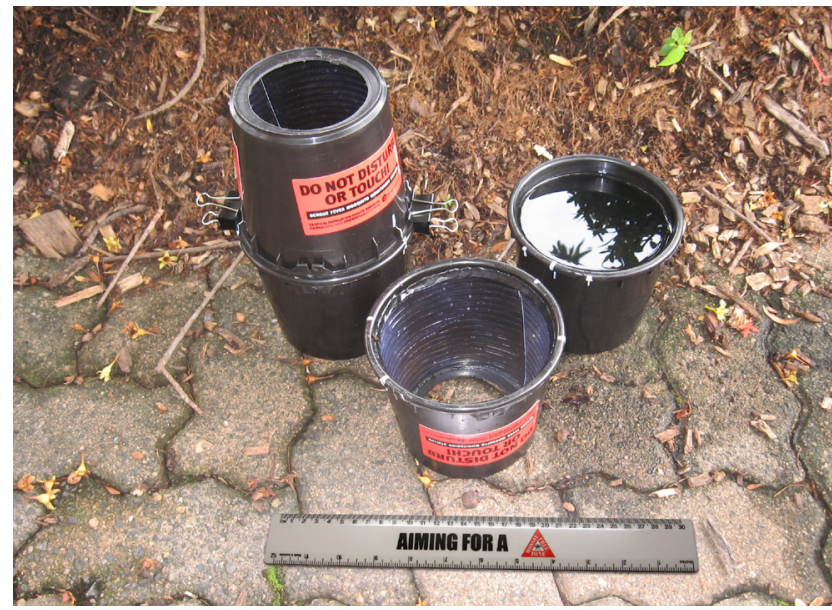

Figure 1. The components of the double sticky trap (DST): black buckets with panels of glue, and a fully assembled SDT with holding clips and panels.

ovitrap, one ST, and one DST were placed at ground level in the enclosed porch area to protect them from animals, wind, rain, and direct sunlight. All houses used in St. Augustine and in Tamana were stand-alone houses with a yard that afforded suitable locations for trap deployment.

All ovitraps were serviced weekly, as described by Chadee et al. (1995). Ovitraps were exposed for one week at a time for a total of ten weeks. Each week, paddles labeled with the house number and location were removed and replaced with egg-free paddles, the water in each ovitrap discarded, number of immatures counted and collected, ovipot scrubbed to remove any eggs laid or attached to the inside of the ovitraps (Chadee et al. 1995), and 350 $\mathrm{ml}$ of fresh tap water added. The handling of paddles and identification of eggs and immatures after collection were described by Chadee (2009) using standard taxonomic keys (Darsie and Ward 1981).

The ST and DSTs were serviced according to Ritchie et al. (2003) and Williams et al. (2006). All adult mosquitoes and immatures collected in the two types of sticky traps were counted and all live adults were collected using fine tip forceps and placed into labeled (trap types and house number) tubes containing alcohol. All immatures were also collected in labeled tubes and transported to the Parasitology Laboratory, Department of Life Sciences, University of the West Indies, St. Augustine, Trinidad. At the laboratory, immatures and adults were identified according to species using standard keys for container breeding mosquitoes (Darsie and Ward 1981).

The results of this study were subjected to an analysis of variance to determine differences in the sensitivity and specificity of the three traps and to determine any location or position effects. The differences among the three traps were further analyzed by transforming the data into a contingency table and running a G-test (Sokal and Rohlf 1981). In addition, the distribution of positive traps among the ten houses was analyzed using a Kendell co-efficient of Rank correlation test (Sokal and Rohlf 1981). 
Table 1. Total number of adult and immature Ae, aegypti collected by sticky and standard ovitraps in Tamana, Trinidad, West Indies (2008).

\begin{tabular}{|c|c|c|c|c|c|c|}
\hline \multirow[t]{2}{*}{ Weeks } & \multicolumn{2}{|c|}{ Sticky trap } & \multicolumn{2}{|c|}{ Double sticky trap } & \multicolumn{2}{|c|}{ Ovitrap } \\
\hline & Adults & Immatures & Adults & Immatures & Eggs & Larvae \\
\hline 1 & 16 & $\begin{array}{l}145^{\star} \\
182^{\star \star}\end{array}$ & 31 & $\begin{array}{l}170^{*} \\
139 * *\end{array}$ & 1 & 0 \\
\hline 2 & 41 & $61^{* *}$ & 28 & $18^{\star \star}$ & 47 & 9 \\
\hline 3 & 25 & $202^{* *}$ & 45 & $260^{* *}$ & 56 & 4 \\
\hline 4 & 28 & $36^{* *}$ & 26 & $25^{\star \star}$ & 56 & 0 \\
\hline 5 & 15 & $350^{* *}$ & 19 & $375^{\star *}$ & 101 & 69 \\
\hline 6 & 12 & $350^{\star *}$ & 24 & $153^{\star \star}$ & 49 & 40 \\
\hline 7 & 13 & $101^{\star *}$ & 27 & $49^{* *}$ & 41 & 2 \\
\hline 8 & 14 & $97^{\star *}$ & 39 & $101^{\star *}$ & 62 & 14 \\
\hline 9 & 23 & $47^{\star *}$ & 41 & $201^{* *}$ & 53 & 23 \\
\hline$` 10$ & 23 & $21^{\star *}$ & 36 & $163^{\star *}$ & 51 & 19 \\
\hline Total & 220 & 1,592 & 316 & 1,652 & 517 & 180 \\
\hline
\end{tabular}

${ }^{\star}$ Culex quinquefasciatus immatures; ${ }^{* *}$ Aedes aegypti immatures.

Table 2. The total number of adult and immature Ae. aegypti collected by sticky and standard ovitraps in St. Augustine, Trinidad, West Indies (2008).

\begin{tabular}{ccccccc} 
Weeks & \multicolumn{2}{c}{ Sticky trap } & \multicolumn{2}{c}{ Double sticky trap } & \multicolumn{2}{c}{ Ovitrap } \\
\hline & Adults & Immatures & Adults & Immatures & Eggs & Larvae \\
\cline { 2 - 7 } 1 & 149 & 425 & 207 & 424 & 241 & 0 \\
2 & 97 & 1,075 & 100 & 655 & 449 & 9 \\
3 & 132 & 1,559 & 125 & 893 & 269 & 0 \\
4 & 184 & 421 & 104 & 795 & 199 & 3 \\
5 & 127 & 255 & 91 & 846 & 116 & 0 \\
6 & 236 & 421 & 341 & 951 & 463 & 0 \\
7 & 193 & 503 & 321 & 771 & 391 & 1 \\
8 & 101 & 606 & 333 & 873 & 401 & 0 \\
9 & 197 & 590 & 319 & 799 & 385 & 0 \\
10 & 199 & 485 & 345 & 790 & 361 & 0 \\
\hline Total & $\mathbf{1 , 4 8 0}$ & $\mathbf{5 , 9 0 0}$ & $\mathbf{2 , 2 8 6}$ & $\mathbf{7 , 7 7 7}$ & $\mathbf{2 , 7 3 5}$ & $\mathbf{1 3}$ \\
\hline
\end{tabular}

Table 3. Mean (range) number of adult Ae. aegypti collected from the sticky trap, double sticky trap, and ovitraps in Tamana and St. Augustine, Trinidad, West Indies (2009).

Mean number of adults collected in traps

\begin{tabular}{ccc}
\hline Traps & Tamana (Range) & St. Augustine (Range) \\
\hline Sticky & $22(12-41)$ & $148(97-236)$ \\
Double Sticky & $31.6(19-45)$ & $228.6(91-345)$ \\
Ovitraps $^{*}$ & $1.7(1-10)$ & $9.1(4-15)$ \\
\hline
\end{tabular}

*Adults calculated from ovitraps based on 30 eggs per female (Chadee 2009). 


\section{RESULTS}

All three trapping methods successfully collected Ae. aegypti mosquitoes and immature stages (Tables 1 and 2). Other mosquitoes collected in Tamana included 210 Culex quinquefasciatus Say, 167 Limatus durhamii Linn, 12 Ochlorotatus ferox, and ten Wyeomyia species.

From a total of 1,700 Ae. aegypti adults collected in STs in Tamana and St. Augustine, significantly more $(\mathrm{P}<0.01)$ (87\%, 1,480 adults) were collected from St. Augustine than in Tamana (13\%) (Tables 1 and 2). The number of larvae collected in the STs also reflected a similar pattern, with $21 \%(1,592)$ collected in Tamana and 79\% $(5,900)$ collected in St. Augustine.

In Tamana and St. Augustine, the DSTs collected a total of 2,602 Ae. aegypti adults, of which 12\% (316) were collected in Tamana but with significantly more $(\mathrm{P}<0.002)$ $(88 \%, 2,286)$ adults being collected in St. Augustine.

In addition, when the results of the STs and DSTs were compared by transforming the data into contingency tables and subjecting them to a G-test (Sokal and Rohlf 1980), they showed significant differences $(\mathrm{G}=110$; d.f. $1 \mathrm{P}<0.002)$ between the performance of STs and DSTs and between the population sizes of Ae. aegypti collected ( $\mathrm{G}=112.0$; d.f. $1 \mathrm{P}<0.003$ ) in Tamana and St. Augustine (Tables 1 and 2), with significantly more $(\mathrm{P}<0.002)$ adults collected in DSTs than in STs in both Tamana and St. Augustine.

Data from the ovitraps showed a total of 3,252 Ae. aegypti eggs collected from Tamana and St. Augustine but with significantly $(\mathrm{P}<0.002)$ more eggs $85 \%$ or 2,735 eggs collected in St. Augustine compared with $15 \%$ or 517 eggs collected in Tamana. After ten weeks of trapping, 157 of the STs were positive, with 65 in Tamana and 92 in St. Augustine, whereas 169 DSTs were positive with 75 in Tamana and 94 in St. Augustine. With respect to ovitraps, a total of 107 were positive with 44 in Tamana and 63 in St. Augustine.

Table 3 shows the number of Ae. aegypti adults collected in ovitraps, STs, and DSTs in Tamana and St. Augustine. The results also show that STs collected a weekly average of 3.7 and 16.0 Ae. aegypti adults in Tamana and St. Augustine, compared with the DSTs which captured 4.2 and 24.3 adults in Tamana and St. Augustine, respectively. With respect to the ovitrap collections, the average number of eggs collected in Tamana and St. Augustine was 11.8 and 43.4, respectively (Tables 1 and 2).

The efficiencies of the three collection methods are summarized in Table 3. Results from the Kendell co-efficient of rank correlation test indicated that the distributions of eggs and adults in Tamana $(\mathrm{P}=22.0)$ and St. Augustine ( $P$ $=21.5$ ) were random since no significant relationships were detected between the number of eggs and adults captured and position or distribution of traps used in the ten houses at both study sites.

\section{DISCUSSION}

All three trapping methods successfully collected Ae. aegypti and consistently showed the population density was significantly $(\mathrm{P}<0.002)$ greater in urban St. Augustine than in the rural community of Tamana, Trinidad, West Indies. Studies in India (Sharma 1998), Brazil (Hayes et al. 1996, Lourenco-de-Oliveira et al. 2008), Suriname (Tinker 1964), and in Trinidad (Focks and Chadee 1997) showed similar patterns, with lower populations of Ae. aegypti in rural communities than in urban housing centers. In the present study, this pattern was clearly discernable, collecting fewer adults in the ST (3.4 vs 4.2 ), DST (16.0 vs 24.3 ), and also in the ovitrap, fewer eggs collected (11.8 vs 43.4$)$ in rural Tamana than in urban St. Augustine, respectively (Tables 1 and 2).

Several studies have correlated the collections of adults captured in STs and egg counts from ovitraps (Facchinelli et al. 2007, Lourenco-de-Oliveira et al. 2008). However, it is uncertain whether the two collection methods are amenable to such comparisons because ovitraps determine the presence or absence of Ae. aegypti or Aedes albopictus Skuse, while the sticky traps collect gravid females before, during, or after oviposition. It is not surprising that a poor correlation was detected when collections from the ovitraps (Fay and Eliason 1966) and MosquiTRAP were compared in Brazil (Gama et al. 2007), although similar percentages of positive ovitraps and sticky traps were reported in Australia (Ritchie et al. 2003) and in Italy (Facchinelli et al. 2007). It seems questionable whether egg populations should be compared with adult collections because they represent different life stages. Eggs and each immature stage are subject to mortality factors (extrinsic), while each female collected has a fecundity potential of approximately 100 eggs (Clements 1999). The nature of this variability explains why the differences observed in total egg counts in Tamana and St. Augustine (Tables 1 and 2) were significant $(\mathrm{P}<0.002)$; that is, the results showed efficient collections of Ae. aegypti eggs in both Tamana and St. Augustine but with significantly $(\mathrm{P}<0.002)$ more eggs being collected in St. Augustine than in Tamana. However, no correlations were observed between sticky trap (ST and DST) collections and egg counts during the present study, with the number of eggs collected exceeding the number of adults collected at both Tamana and St. Augustine (Tables 1 and 2) and when the data were compared using mean number of adults collected or estimated from the three trapping methods (Table 3 ).

In addition, the distribution of the eggs and adults among the 30 trap sites in Tamana did not differ each week. In fact, the distribution of eggs and adults among the sites appear to be random $(\mathrm{P}=0.22)$ since no significant relationship can be detected between the number of eggs laid or adults collected and the position or distribution of traps used in the ten houses in Tamana. A Kendell co-efficient of rank correlation test (Sokal and Rohlf 1981) was used in both cases. A similar pattern was observed in St. Augustine $(\mathrm{P}=21.5)$, with no significant differences detected among the different traps, position, distribution, and efficiency.

Chadee et al. (1990) and Apostal et al. (1994) reported that gravid Ae. aegypti mosquitoes disperse their eggs over several sites with approximately 11-30 eggs per oviposition container. Therefore the 2,735 eggs collected 
in St. Augustine may represent 91.2 females (30 eggs per female), whereas the 516 eggs collected in Tamana may represent the oviposition of 17 Ae. aegypti females. When these numbers of adults are compared with collections from both the ST and DSTs, the sensitivity of these traps is far superior to ovitraps, with 1,480 and 2,286 adults collected in St. Augustine, respectively (Tables 1 and 2). The fact that all traps were exposed and collected each week at the St. Augustine study site, for example, indicates that more females may have visited these traps than previously considered, with averages of 148, 228.6, and 91 collected per week in single sticky, double sticky traps and ovitraps, respectively (see Table 4). In fact, collections from this study far exceeded collections from similar sticky trap studies in Italy with 83 females (Facchinelli et al. 2007), 31 out of 401 marked females in Mexico (Ordonez-Gonzalez et al. 2001), 0-6 females in Brazil (Lourenco-de-Oliveira et al. 2008), and a mean of 6.0 females per sticky trap in Australia (Ritchie et al. 2003).

The collection of large numbers of adults and immature stages in both the ST and DSTs has afforded the opportunity to support the incorporation of larvicidal ingredients in the ST and DSTs with $>1,500$ immatures collected in Tamana and $>6,000$ immatures collected in St. Augustine (Tables 1 and 2). The utilization of larvicides incorporated into the water used in these sticky traps may serve to destroy the immature stages should traps be missed by surveillance workers and thus become point sources for producing large numbers of adults. Methoprene pellets, which do not impact Ae. aegypti oviposition in ovitraps (Ritchie and Long 2003 ), are currently used by Queensland Health to prevent mosquito production in sticky ovitraps. These results also support the "lure and kill" concept currently being used in the design of new and improved lethal ovitraps (Ritchie et al. 2009). The collection of adults from sticky traps can also be incorporated into xenomonitoring protocols for testing adult Ae. aegypti for dengue viruses (Bang et al. 2001), for insecticide resistance studies (Rodriguez et al. 2001), for reducing adult mosquito populations, that is, reducing manvector contact within houses (Ritchie et al. 2003, Rapley et al. 2009), and for surveillance and epidemiological studies (Ritchie et. al. 2003).

In summary the results of this study suggest that DSTs collected significantly $(\mathrm{P}<0.02)$ more Ae. aegypti in both Tamana and St. Augustine and are suitable alternatives to the modified ovitraps which are currently being used in the Caribbean region. The fact that adult mosquitoes are collected allow workers not only to remove large numbers of adults but also to use the captured dead adults for molecular studies, while the adults that are alive (taken when servicing the traps) can be processed for virus isolation or for further molecular studies.

\section{Acknowledgments}

We thank Messrs. Dexter Maraj and Barry D. Samlalsingh for assistance in the field and Dr. Joan Sutherland for assistance in the preparation of the manuscript.

\section{REFERENCES CITED}

Apostal, B.I., W.C. Black, P. Reiter, and B.R. Miller. 1994. Use of randomly amplified polymorphic DNA amplified by polymerase chain reaction markers to estimate the number of Aedes aegypti families at oviposition sites in San Juan, Puerto Rico. Am. J. Trop. Med. Hyg. 51: 8997.

Bang, M.J., R. Tan, E. Listiyamingsih, B.H. Kay, and K.R. Porter. 2001. Detection of dengue viral RNA in Aedes aegypti (Diptera: Culicidae) exposed to sticky lures using reverse-transcriptase polymerase chain reaction. J. Med. Entomol. 38: 720-724.

Buxton, P. and G. Hopkins. 1927. Researches in Polynesia and Melanesia. Part I-V. London School of Hygiene and Tropical Medicine, London, pp. 1-127.

Chadee, D.D. 2009. Oviposition strategies adopted by gravis Aedes aegypti (L.) (Diptera: Culicidae) as detected by ovitraps in Trinidad, West Indies (202-2006). Acta Tropica 111: 279-283.

Chadee, D.D. 2010. Emergency control of dengue fever in the Americas, In: L.V. Berhardt (ed.) Adv. Med. Biol. 3: Nova Science Publications, New York. (in press)

Chadee, D.D. and P.S. Corbet. 1987. Seasonal incidence and diel patterns of oviposition in the field of the mosquito Aedes aegypti (L.) (Diptera: Culicidae) in Trinidad, West Indies: a preliminary study. Ann. Trop. Med. Parasitol. 81: 151-161.

Chadee, D.D., P.S. Corbet, and J.J.D. Greenwood. 1990. Egglaying yellow fever mosquito avoid sites containing eggs laid by themselves or by conspecifics. Entomol. Exp. Appl. 57: 295-298.

Chadee, D.D., P.S. Corbet, and H. Talbot. 1995. Proportions of eggs laid by Aedes aegypti on different substrates with an ovitrap in Trinidad, West Indies. Med. Vet. Entomol. 9: 66-70.

Chadee, D.D., A. Lakhan, W.R. Ramdath, and R.C. Persad. 1993. Oviposition response of Aedes aegypti mosquitoes to different concentrations of hay infusion in Trinidad, West Indies. J. Am. Mosq. Contr. Assoc. 9: 346-348.

Clements, A.N. 1999. The Biology of Mosquitoes. Vol. 2. Wallingford, Oxon, CABI Publishing.

Darsie, R.F. and R.A. Ward. 1981. Identification and geographical distribution of the mosquitoes of North America, North of Mexico. Mosq. Syst. Supplement 1: $1-313$.

Facchinelli, L., L. Valerio, M. Pombi, P. Reiter, C. Costantini, and A. Della Torre. 2007. Development of a novel sticky trap for container-breeding mosquitoes and evaluation of its sampling properties to monitor urban populations of Aedes albopictus. Med. Vet. Entomol. 21: 183-195.

Fay, R.W. and D.A. Eliason. 1966. A preferred oviposition site as a surveillance method for Aedes aegypti. Mosq. News 26: 531-535

Focks, D.A. and D.D. Chadee. 1997. Pupal survey: An 
epidemiologically significant surveillance method for Aedes aegypti. An example using data from Trinidad, West Indies. Am. J. Trop Med. Hyg. 56: 159-167.

Gama, R.A., E.M. Silva, I.M. Silva, M.C. Resende, and A.E. Eiras. 2007. Evaluation of the sticky MosquiTrap for detecting Aedes (Stegomyia) aegypti (L.) (Diptera: Culicidae) during the dry season in Belo Horizonte, Minas Gerais, Brazil. Neotrop. Entomol. 36: 294-302.

Gjullin, C.M, J.O. Johnson, and F.W. Plapp. 1965. The effects of odours released by various waters on the oviposition sites selected by two species of Culex. Mosq News 25: 268-272.

Hayes, C.G., I.A. Phillips, J.D. Callahan, W.F. Griebernow, K.C. Hyams, S. Jue-Wu, and D.M. Watts. 1996. The epidemiology of dengue virus infection among urban jungle, and rural populations in the Amazon region of Peru. Am. J. Trop. Med. Hyg. 55: 459-463.

Hazard, E.I., M.S. Mayer, and K.E. Savage. 1967. Attraction and oviposition stimulation of gravid female mosquitoes by bacteria isolated from hay infusion. Mosq. News 27: 133-136.

Lourenco-de Oliveira, R., J.B.P. Lima, R. Peres, F. Da Casta Alves, A.E. Eiras and C.T. Codeco. 2008. Comparison of different uses of adult traps and ovitraps for assessing dengue vector infestation in endemic areas. J. Am. Mosq. Contr. Assoc. 24: 387-392.

Manefield, T. 1951. Investigations of the preferences shown by Aedes (Stegomyia) aegypti Linn and Culex (Culex) fatigans Wied. for specific types of breeding water. Proc. Linn. Soc. 76: 149-154.

McCall, P.J., G. Harding, J. Roberts, and B. Auty. 1996. Attraction and trapping of Aedes aegypti (Diptera: Culicidae) with host odors in the laboratory. J. Med. Entomol. 33: 177-179.

Muir, L E. and B.H. Kay. 1998. Aedes aegypti survival and dispersal estimated by mark-release-recapture in northern Australia. Am. J. Trop. Med. Hyg. 58: 277282.

O'Gower, A.K. 1963. Environmental stimuli and the oviposition behaviour of Aedes aegypti var. queenslandensis Theobald (Diptera: Culicidae). Anim. Behav. 2: 189-197.

Ordonez Gonzalez, J. G., R. Mercado-Hernandez, A.E. Flores-Suarez, and I. Fernández- Salas. 2001. The use of sticky ovitraps to estimate dispersal of Aedes aegypti in northeastern Mexico. J. Am. Mosq. Contr. Assoc. 17: 93-97.

PAHO, 1994. Dengue and Dengue Haemorrhagic Fever in the Americas: Guidelines for Prevention and Control. Washington D.C. Pan Am. Hlth. Org.

Rapley, L.P., P.H. Johnson, C.R. Williams, R.M. Silcock, M. Larkman, S.A. Long, R.C. Russell, and S.A. Ritchie. 2009. A lethal ovitrap-based mass trapping scheme for dengue control in Australia: II. Impact on populations of the mosquito Aedes aegypti. Med. Vet. Entomol. 23: 303-316.

Reiter, P., M.A. Amador, and N. Colon. 1991. Enhancement of the CDC ovitrap with hay infusion for daily monitoring of Aedes aegypti populations. J. Am. Mosq. Contr. Assoc. 7: 52-55.

Ritchie, S.A. 2001. Effect of some animal feeds and oviposition substrates on Aedes oviposition in ovitraps in Cairns, Australia. J. Am. Mosq. Contr. Assoc. 17: 206-208.

Ritchie, S. and S. Long. 2003. Does S-methoprene effect oviposition by Aedes aegypti in an ovitrap? J. Am. Mosq. Contr. Assoc. 19: 170-171.

Ritchie, S.A., S. Long, G. Smith, A. Pyke, and T. Knox. 2003. Entomological investigations in a focus of dengue transmission in Cairns, Queensland, Australia using the sticky ovitrap. J. Med. Entomol. 41: 1-4.

Ritchie, S.A., L.P. Rapley, C. Williams, P.H. Johnson, M. Larkman, R.M. Silcock, S.A. Long, and R.C. Russell. 2009. A lethal ovitrap-based mass trapping scheme for dengue control in Australia: I. Public acceptability and performance of lethal ovitraps. Med. Vet. Entomol. 23: 295-302.

Rodríguez, M.M., J. Bisset, D.M. de Fernandez, L Lauzan, and A. Soca. 2001. Detection of insecticide resistance in Aedes aegypti (Diptera: Culicidae) from Cuba. J. Med. Entomol. 38: 623-628.

Santana, A.L., R.A. Roque, and A.E. Eiras. 2006. Characteristics of grass infusions as ovipositing attractants to Aedes (Stegomyia) (Diptera: Culicidae). J. Med. Entomol. 43: 214-220.

Sharma, S.K. 1998. Entomological investigations of DF/ DHF outbreak in rural areas of Hissar District, Haryana, India. Deng. Bull. 22: 36-38.

Sokal, R.R. and F.J. Rohlf. 1981. Biometry, The Principles and Practice of Statistics in Biological Research, ( $2^{\text {nd }}$ ed). New York, W.H. Freeman.

Tinker, M.E. 1964. Aedes aegypti larval habitats in Surinam. Pan Am. Hlth. Org. Bull.8: 293-301

Trexler, J.D., C.S. Apperson, and C. Schal. 1998. Laboratory and field evaluations of oviposition responses of Aedes albopictus and Aedes triseriatus (Diptera: Culicidae) to oak leaf infusion. J. Med. Entomol. 35: 967-975.

Williams, C.R., S.A. Ritchie, R.C. Russell, M. Geier, and S.A. Long. 2006. Field efficacy of the BG-Sentinel compared with the CDC backpack aspirator and $\mathrm{CO}_{2}$ baited EVS trap for collection of adult Aedes aegypti in Cairns, Queensland, Australia. J. Am. Mosq. Contr. Assoc. 22: 296-300.

Zeichner, B.C. and M.J. Perich. 1999. Laboratory testing of a lethal ovitrap for Aedes aegypti. Med.Vet. Entomol. 13: 234-238. 